profession to understand the impact of communication and information technologies on the provision of health care. Some sections of the profession are already actively experimenting with the technology. However, the implications of the Internet extend far beyond the technology itself. We should now, and with some haste, be examining its implications for the future of medical practice.

ENRICO COIERA ewc@hplb.hpl.hp.com Senior project manager

Hewlett-Packard Research Laboratories, Stoke-Gifford,

Bristol BS12 6QZ
1 Coiera E. Medical informatics. $B M \mp$ 1995;310:1381-7.

2 Weale S., Surfer, heal thyself. Guardian 1995 Sept 20:13.

3 Fridsma DB, Ford P, Altman R. A survey of patient access to electronic mail: attitudes, barriers and opportunities. Proceedings of the Symposium on Computer Applications in Medicine. fournal of the American Medical Informatics Association (symposium supplement) 1994:15-9.

4 Home page: http://www.bmj.com/bmi/

5 Sellu D. Clinical encounters in cyberspace. BMF 1996;312:49.

6 Anderson R. NHS-wide networking and patient confidentiality. $B M 7$ 1995;311:5-6.

7 Klein R. Priorities and rationing:pragmatism or principles? $B M F$ 1995:311:761-2.

8 Ham C. Health care rationing. $B M \mathcal{F} 1994 ; 310: 1483-4$.

9 Bolam v Friern Barnet HMC 19571 WRL 582.

9 Bolam v Friern Barnet HMC 19571 WRL 582.

1 Doctors in the dock. Economist 1995 Aug 19:23-4.

1 Gurwitz B. Clinical guidelines and the law. BMF 1995:311:2.

3 LaPorte RE, Marler E, Akazawa S, Sauer F, Gamboa C, Shenton C, et al. The death of biomedical journals. BMf 1995;310:1387-9.

14 Home page: http://omni.nott.ac.uk

15 LaPorte RE. Global public health and the information superhighway. BMf 1994;308:1651-2.

\title{
Virtual reality in rehabilitation
}

\section{Potential benefits for people with disability or phobias}

Virtual reality is an interactive, computer generated environment that simulates the real world. Accessible via an interface that has been adapted to the human senses, it provokes in the user the sensation of immersion, like Alice in Wonderland crawling down the rabbit hole and finding herself in a new fantastic world. ${ }^{1}$ A dataglove wired with fibreoptics to record the degree of bending of fingers and wrists, a head-mounted display, and an exoskeleton surrounding the user's limbs to give feedback, project the user into the virtual environment and report back on his or her actions within it. The user can react intuitively to the virtual world, any actions being detected by position and movement sensors placed strategically on his or her body. A computer calculates the changes occurring in the virtual world according to the rules chosen by its creator and feeds the results back to the user. Applications appeal to disabled and non-disabled persons alike ${ }^{2}$ : telepresence, for example, allows specialists to operate on patients in remote hospitals. Three applications of immersive virtual reality in rehabilitation, ${ }^{3}$ two of them therapeutic, are discussed below:

Physically disabled students are often excluded from participating in laboratory experiments because of their reduced mobility and manual capabilities or the risk that they might lose control when in a hazardous environment. A virtual laboratory for disabled students has been studied. ${ }^{4}$ Because the interface has to meet the special needs of the user, the researchers selected a relatively homogenous population. The students all had spinal cord injuries ranging from $\mathrm{C} 2$ to $\mathrm{T} 1$ and were of at least average intelligence and emotional stability. The objects in the virtual laboratory included a representation of a traditional laboratory, with symbols representing information and illustrating principles of physics, and a virtual vehicle that allows users to experience the physical phenomena under investigation, such as the acceleration created by a force. The students encountered few problems acting within the virtual world, but some were not able to put on the equipment independently or adjust it over time to avoid painful pressure points.

Acrophobia or fear of heights is another condition in which virtual reality may have a role. Rothbaum et $a \bar{l}$ performed a controlled trial of graded exposure to heights using virtual reality. Sufferers were randomly assigned either to treatment or to a waiting list. The treatment group had seven weekly 35-45 minute sessions on virtual balconies, high bridges, or in the glass elevator of a skyscraper. A therapist watched and commented on the exposure, during which the patient's subjective discomfort was monitored. Before and after treatment, anxiety, avoidance, distress, and attitudes were measured using standard questionnaires. The results were comparable to those of standard therapy with stepwise exposure to real, frightening but sustainable stimuli. However, there was no comparison group on conventional treatment, no follow up data, and the numbers were small. Also the subjects were volunteers, rather than people actively seeking treatment.

The third application is akinesia or freezing gait in Parkinson's disease. This debilitating symptom is characterised by progressive shortening of the patient's stride and ultimately the inability to move forward at all. Treatment with drugs is marred by on-off effects and side effects such as dyskinesia. However, patients with akinesia can walk over objects or through doorways with little effort, an effect known as kinesia paradoxa. Using a virtual reality technique called augmented reality, Weghorst et al projected virtual objects on to the patients' physical world to give them the impression that they were walking over or through them, thereby restoring their mobility. ${ }^{6}$ They suggested various aproaches to mimic steady objects on the ground while the patient is moving. However, existing visual displays are not bright enough to compete with ambient light, while severe akinesia demands the creation of a highly realistic and static representation of the obstacle. Dyskinesia, too, was found to respond to augmented reality, suggesting a more complex mechanism underlying kinesia paradoxa.

Using virtual reality techniques in rehabilitation raises ethical considerations, especially when mentally challenged or very young people are involved. ${ }^{7}$ (In the examples given above, all the patients gave informed consent.) In addition, virtual reality could have undesirable side effects due to equipment failure, fatigue, or motion sickness. ${ }^{89}$ It could also cause unintended changes in the patient's attitude and behaviour, worsen existing difficulty in distingishing between reality and delusion (for example in patients with schizophrenia), or cause distress from the virtual experiences themselves.

The applications described above exemplify how virtual reality can help even severely disabled people to participate and contribute safely even in hazardous and complex tasks. The new technology can provide corrective experiences that can ameliorate attitudes and anxieties. It could also yield new insights into disease mechanisms while providing ingenious and effective tools to re-enable handicapped people. Many more examples could be cited, ${ }^{1-46}$ some of which will be presented at a conference on medicine and virtual reality in 
San Diego later this month. To reduce the cost, the optical resolution and complexity of the environment can be kept low in most rehabilitation applications. The new technology offers great potential benefit in rehabilitation but remains to be properly explored and evaluated.

MICHAEL HENNING ANDREAE Research student

Bagrit Centre for Biological and Medical Systems, Imperial College of Science, Technology and Medicine, London SW7 2BX
1 Pimentel K. Virtual reality through the new looking glass 2nd rev ed. New York: McGrawHill, 1995. 2 Loeffler C, ed. Virtual reality casebook. New York: Van Nostrand, 1994.

3 Greenleaf WJ, Tovar MA. Augmenting reality in rehabilitation medicine. Artificial Intelligence in Medicine 1994;6:289-99.

4 Nemire $K$, Burke A, Jacoby R. Human factors engineering of a virtual laboratory for students with disabilities. Presence 1994;3:216-26.

5 Rothbaum B, Hodges LF, Kooper R, Opdyke D, Williford JS. Effectiveness of computer-generated (virtual reality) graded exposure in the treatment of acrophobia. Am $\mathcal{f}$ Psychiatry 1995;152:626-8.

6 Weghorst SW, Prothero J, Furness T, Anson D, Riess T. Virtual images in the treatment of Parkinson's disease akinesia. In: Morgan K, Satava RM, Sieburg HB, Matheus R, Christensens JP, eds. Medicine meets virtual reality II. 1995;30:242-3.

7 Being and believing: ethics of virtual reality [editorial]. Lancet 1991;338:283-4.

7 Being and believing: ethics of virtual reality [editorial]. Lancet 1991;338:283-4.
8 Mon-Williams M, Wann JP, Rushton S. Binocular vision in a virtual world: visual deficits following the wearing of a head-mounted display. Ophthalmic Physiol Opt 1993;13:387-91.

9 Regan EC, Price KR. The frequency of occurrence and severity of side-effects of immersion virtual reality. Aviation, Space, and Environmental Medicine 1994;65:527-30.

\section{Minimally invasive surgery for male subfertility}

\section{Giving hope to azoospermic men}

Minimally invasive surgery is well established in the diagnosis and treatment of infertile women. With continuing advances in the design of fibreoptic instruments, especially the development of small diameter endoscopes, the concept of minimally invasive surgery can now be applied to the diagnosis and treatment of male subfertility, particularly in the management of azoospermic men. ${ }^{2}$

Azoospermia is caused either by obstruction or absence of the vas deferens or by abnormalities in spermatogenesis. Obstructive azoospermia - due to bilateral congenital absence of the vas deferens, a failed reversal of vasectomy, or inflammatory epididymitis-is the reason for infertility in $1-2 \%$ of infertile couples. ${ }^{3}$ Until recently, azoospermic men were considered to be irrevocably sterile, with their only option being donor insemination. It is now clear that spermatozoal transit through the epididymis is not crucial for fertilisation as pregnancies have occurred after epididymovasostomy $y^{4}$ and through conventional in vitro fertilisation with surgically retrieved epididymal spermatozoa. ${ }^{5}$ Obstructive azoospermia has been successfully treated by open microsurgical reconstruction, with $88 \%$ of cases in one series yielding quantities of spermatozoa in the ejaculate that were considered to be sufficient for either spontaneous pregnancy or in vitro fertilisation. ${ }^{6}$

In men with inoperable vasal obstruction or abnormal spermatogenesis the aim of treatment is to recover enough motile spermatozoa for use in conventional in vitro fertilisation. One option is open microepididymal sperm aspiration. ${ }^{\text {? }}$ The resulting spermatozoa, however, are often of poor quality with poor general and forward motility and low density. ${ }^{8}$ Until recently this meant that rates of fertilisation and pregnancy after open aspiration were disappointingly low, at $11 \cdot 2 \%$ and $8 \cdot 7 \%$ respectively. The recent introduction of intracytoplasmic sperm injection has, however, revolutionised treatment. ${ }^{9}$ This technique involves the injection of a single viable spermatozoon into the cytoplasm of an oocyte in metaphase II. It requires only a few viable spermatozoa to achieve fertilisation..$^{10}$ In a retrospective comparative study in 1994, Silber and colleagues showed that intracytoplasmic sperm injection with sperm retrieved by open microepididymal aspiration resulted in rates of pregnancy and delivery that were not significantly different from rates achieved by men with normal sperm counts. ${ }^{11}$

The drawback of open microsurgical retrieval of sperm is that it requires a general anaesthetic and surgical exploration of the scrotum. The procedure also requires extensive training and expertise and is currently available in only a few specialised fertility centres. Potential complications include pain, haematoma of the scrotum, and infection, and in our experience few men agree to undergo the procedure twice.

By contrast, a new minimally invasive technique recently described by Craft et $a l^{12}$ can be performed under sedation. Percutaneous epididymal sperm aspiration entails the insertion of a 22 gauge butterfly needle into the epididymis, after which negative pressure is generated by a $20 \mathrm{ml}$ syringe connected to the plastic tubing of the butterfly needle. Scrotal exploration is not needed, and the procedure carries minimal risk of complications. The only reported complaint in 38 men who underwent the procedure during the study period was dull testicular pain, which is easily controlled by oral analgesics. In men with failed reversal of vasectomy, bilateral congenital absence of the vas deferens, inflammatory vasal obstruction, or partial testicular failure the procedure achieved a mean fertilisation rate of $32 \cdot 7 \%$ and a rate of pregnancy per embryo transfer procedure of $28.6 \%$. The rate of pregnancy is similar to that after open microepididymal sperm aspiration, and similar to that in normal fertile men provided viable spermatozoa are retrieved for microinjection. ${ }^{12}$ Data on rates of live birth are not yet available.

Methods for extracting sperm from the testicle have been similarly revolutionised by minimally invasive techniques. Percutaneous testicular sperm aspiration has removed the need for open testicular biopsy. ${ }^{13}$ The technique has resulted in positive pregnancy tests in partners of azoospermic men with or without vasal or epididymal obstruction. The chances of aspirating testicular sperm are greatest if there is active spermatogenesis in the seminiferous tubules. If this is not present open testicular biopsy may still be necessary.

Minimally invasive surgery is transforming the prospects of fertility for azoospermic men. Furthermore, techniques for transporting gametes from the site of collection to a central embryology laboratory, where intracytoplasmic sperm injection and related procedures are performed, will make these complex therapeutic procedures more accessible. ${ }^{14}$

Y KHALIFA

Clinical research fellow in reproductive medicine J G GRUDZINSKAS Professor of obstetrics and gynaecology

Joint Academic Unit of Obstetrics, Gynaecology,

and Reproductive Physiology,

London Hospital Medical College,

London E1 2AD

1 Craft I, Shrivastav P. Treatment of male infertility. Lancet 1994;344:191-2.

2 Craft II, Tsirigotis M, Bennet V, Taranissi M, Khalifa Y, Hogewind G, et al. Percutaneous epididymal sperm aspiration and intracytoplasmic sperm injection in the management of infertility due to obstructive azoospermia. Fertil Steril 1995;63:1038-42.

3 Hull MGR., Glazener CMA, Kelly NJ, Conway DI, Fosters PA, Hinton RA, et al. Population study of causes, treatment and outcome of infertility. $B M F$ 1985;291:1693-7.

4 Hanley HG. The surgery of male subfertility. Ann R Coll Surg Engl 1955;17:159-83. 